\title{
REPLICATION OF GAMMA-IRRADIATED MATERIALS IN SUSEN HOT CELLS
}

\author{
Mariia ZIMINA, Jiří PROCHÁZKA, Jan PATERA, Ondřej LIBERA \\ Research Centre Řež, Husinec, Czech Republic, EU, \\ mariia.zimina@.cvrez.cz
}

https://doi.org/10.37904/metal.2019.714

\begin{abstract}
The work is designed for the establishment of experimental techniques for replicating of highly-active neutron irradiated samples in SUSEN hot-cells. Using the most sensitive devices for dimensions and defects measurements of irradiated materials is limited due to the negative effect on their operation lifetime and accuracy. Therefore, new replication techniques are needed for the long time operation of the hot cells and its equipment which are sensitive to gamma radiation. The replicas of irradiated material, that are made using manipulators inside the hot-cell, are made of the polymers that are not activated during the operation, so that they can be easily used for the standard measurement procedures. This work describes 3D dimensions measurement and reconstruction of the irradiated materials and evolution of the replication methodology.
\end{abstract}

Keywords: Nuclear materials, characterization, testing methods

\section{INTRODUCTION}

The work was designed to establish the procedure of the accurate measurement of the dimensions of highlyactive neutron irradiated samples in SUSEN hot-cells. In order to gain the sensitive information about the dimensional changes of the materials after any procedure, precise measurement devices are used. However, these devices are sensitive to gamma radiation produced e.g. by the materials from the nuclear reactors $[1,2]$. Thus, the use of replicas of samples and surfaces is required to prolong the lifetime of the measuring machines. Moreover, replicas allow measuring of highly activated materials that cannot otherwise be measured because of a severe damage to the machine or its destruction with no obtained results. The idea of replication is presented in the scheme in Figure 1.

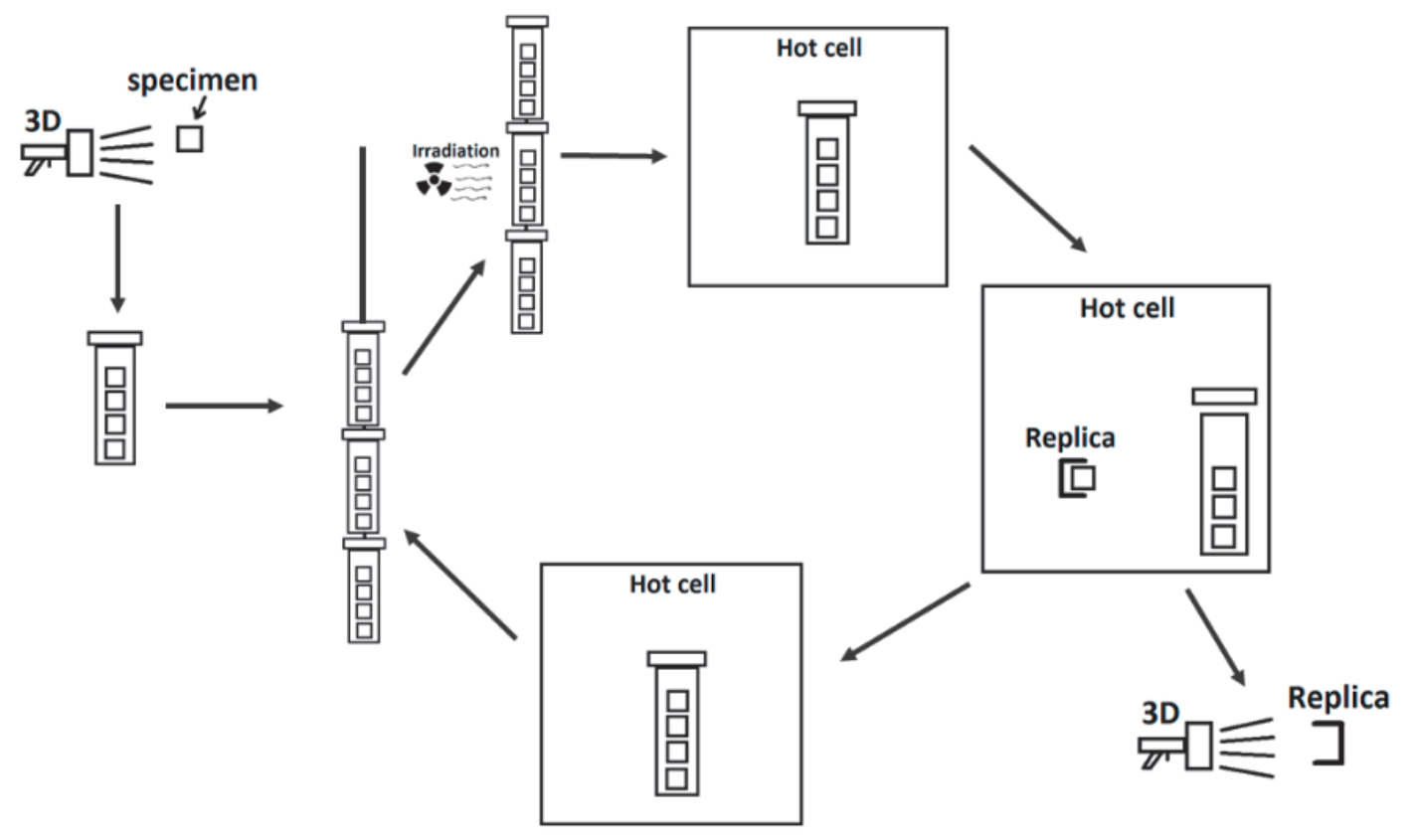

Figure 1 The idea of replication of irradiated materials 
Our research team has developed the mechanical system for replication of highly activated materials using manipulators inside the hot cell. It allows the use of 3D measuring devices, where the measurement is performed outside the hot-cell on produced non-active replicas.

This paper describes the technique and shows several examples of its application.

\section{TECHNIQUE DEVELOPEMENT}

For the establishment of the proper methodology of the replication technique on neutron irradiated materials, first, the selection of suitable replication materials / matters was made. The second stage was irradiation of different replication materials, using SUSEN gamma-irradiation facility, and a comparison of their properties. After all, the replication system for hot-cell application using mechanical manipulators was developed, manufactured and tested. For the method evaluation, the measurement of inactive samples using two types of 3D measurement device (3D laser scanning profilometry) was performed.

\section{RESULTS}

\subsection{Replication matter selection}

Five commercially available polymer matters were gamma-irradiated up to the dose of $2.3 \mathrm{kGy}$ and rate 1.1 $\mathrm{kG} / \mathrm{h}$. The hardness values, $\mathrm{S}_{\mathrm{A}}$, of the matters were compared prior and after the irradiation using Shore's method by the durometer of type $A$. The best results were shown on only one of the matters, which $S_{A}$ value has changed insignificantly from 26 to 28 after irradiation and no shrinking was observed using 3D scanning method. This material was further used for the replication.

\subsection{Measurement accuracy}

The establishment of the technique requires the understanding of the measurement accuracy. For this aim, the $10 \mathrm{~mm} \times 10 \mathrm{~mm} \times 10 \mathrm{~mm}$ steel cube with ten micro-indents of defined sizes varying from $27 \mu \mathrm{m}$ to $270 \mu \mathrm{m}$ was used. The procedure contained the indentation using micro- and nanoindentation device HYSITRON, the production of the replica using the selected polymer matter and measurements of the indents dimension of both cube and replica surfaces (Figure 2). The resulting deviation between cube's surface and replica is less than $0.002 \mathrm{~mm}$. Furthermore, the accuracy of replica of $\pm 0.008 \mathrm{~mm}$ for artefacts of $0.1-0.2 \mathrm{~mm}$ size was calculated after the repetition of the tests.

\subsection{Measurement accuracy}

The establishment of the technique requires the understanding of the measurement accuracy. For this aim, the $10 \mathrm{~mm} \times 10 \mathrm{~mm} \times 10 \mathrm{~mm}$ steel cube with ten micro-indents of defined sizes varying from $27 \mu \mathrm{m}$ to $270 \mu \mathrm{m}$ was used. The procedure contained the indentation using micro- and nanoindentation device HYSITRON, the production of the replica using the selected polymer matter and measurements of the indents dimension of both cube and replica surfaces (Figure 2). The resulting deviation between cube's surface and replica is less than $0.002 \mathrm{~mm}$. Furthermore, the accuracy of replica of $\pm 0.008 \mathrm{~mm}$ for artefacts of $0.1-0.2 \mathrm{~mm}$ size was calculated after the repetition of the tests.

\subsection{Replication examples}

After the replication methodology was established and accuracy was understood, the replication of the steel cube was performed using the developed manipulation system in the hot cell. The schematic illustration of the system is shown in Figure 3. 
a)

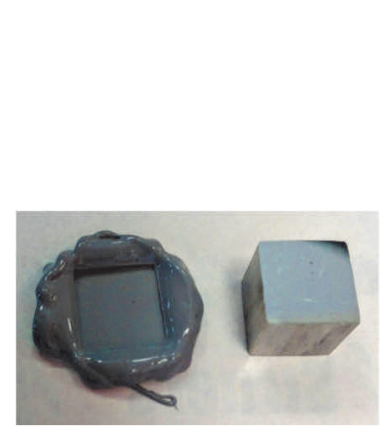

b)

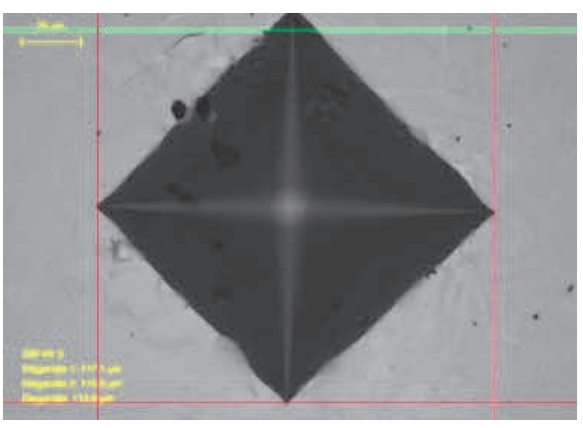

c)

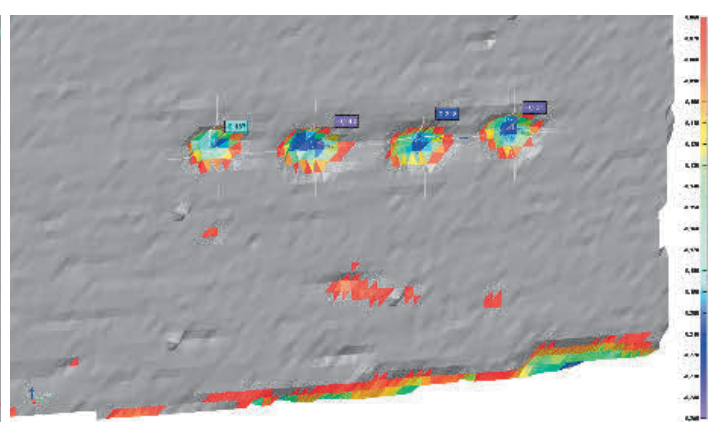

d)
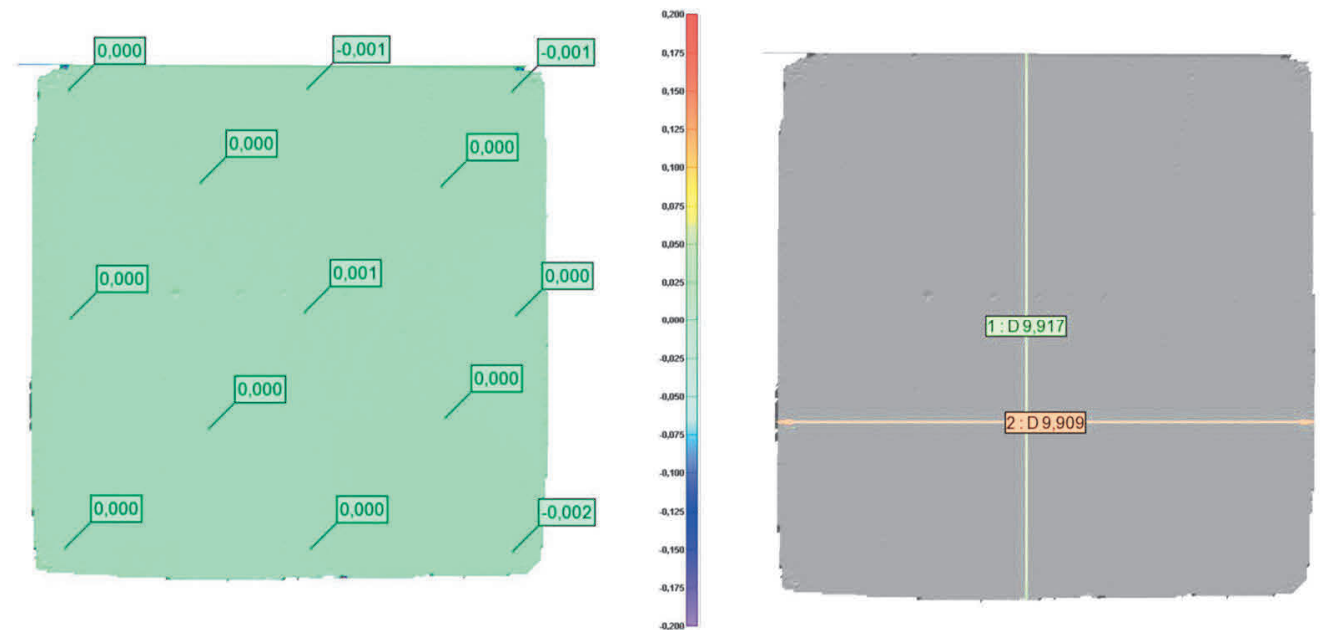

Figure 2 a) Polymer matter replica of the steel cube, b) an example of the indent, c) measurement of the indents sizes using 3D scanner, d) comparison of the result on the cube and replica

\subsection{Measurement accuracy}

The establishment of the technique requires the understanding of the measurement accuracy. For this aim, the $10 \mathrm{~mm} \times 10 \mathrm{~mm} \times 10 \mathrm{~mm}$ steel cube with ten micro-indents of defined sizes varying from $27 \mu \mathrm{m}$ to $270 \mu \mathrm{m}$ was used. The procedure contained the indentation using micro- and nanoindentation device HYSITRON, the production of the replica using the selected polymer matter and measurements of the indents dimension of both cube and replica surfaces (Figure 2). The resulting deviation between cube's surface and replica is less than $0.002 \mathrm{~mm}$. Furthermore, the accuracy of replica of $\pm 0.008 \mathrm{~mm}$ for artefacts of $0.1-0.2 \mathrm{~mm}$ size was calculated after the repetition of the tests.

\subsection{Replication examples}

After the replication methodology was established and accuracy was understood, the replication of the steel cube was performed using the developed manipulation system in the hot cell. The schematic illustration of the system is shown in Figure 3.

Using this mechanical system, the replication of highly irradiated materials can be performed in the hot cell. The inactivated replicas can be then used e.g. for the measurements of the surface topography, roughness and fatigue, and stress-corrosion cracks after the mechanical testing of neutron irradiated materials for nuclear applications. Figure 4a shows the full 3D reconstruction of the cube with defined indents and the comparison with the replica measurement Figure $\mathbf{4 b}$. This reconstruction technique is used for an accurate definition of the volume changes of porous materials after neutron irradiation, e.g. concretes, ceramics or metal foams. 
a)

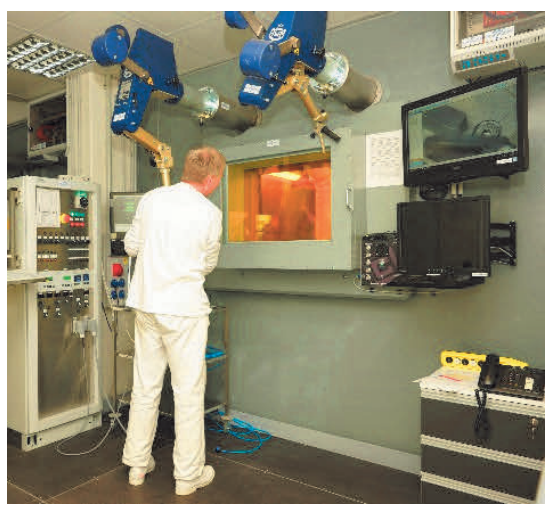

b)

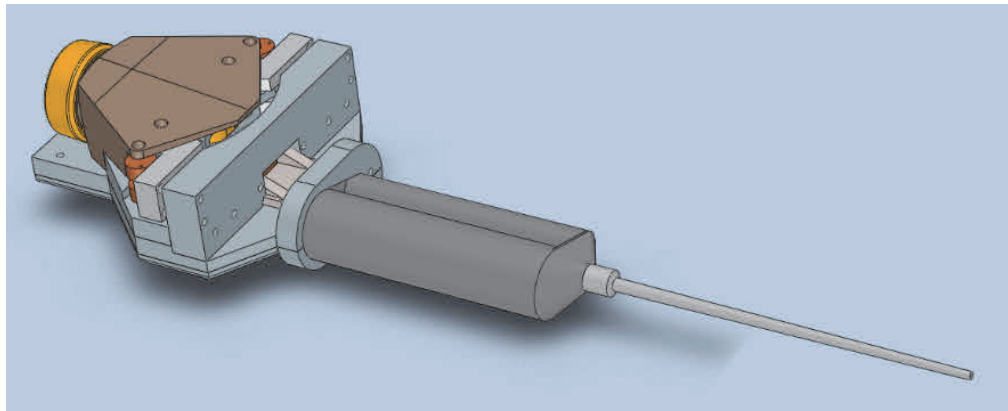

Figure 3 a) The manipulation in the hot cell and b) the replication set developed in CVR

a)

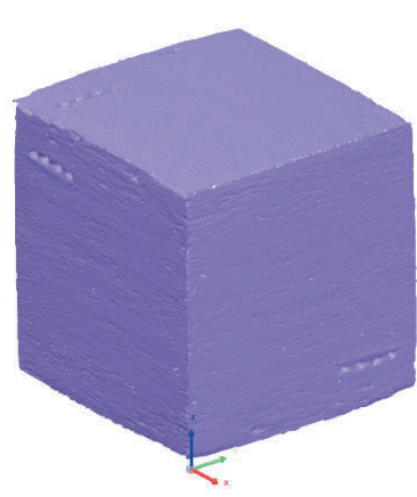

b)

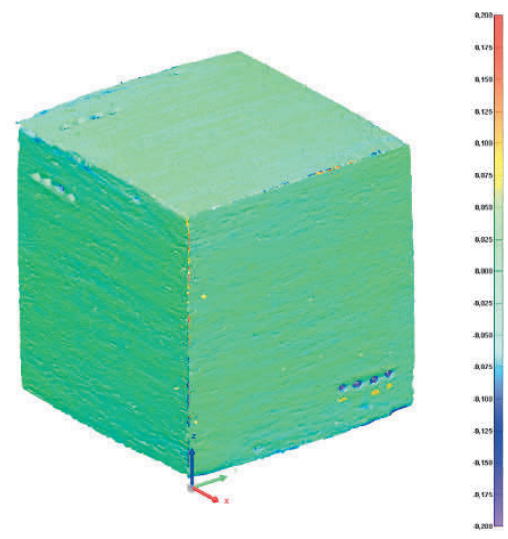

Figure 4 3D reconstruction of a) the steel cube and b) the replicas

Another example of the replication usage is the application during the interrupted mechanical testing of the materials, not only irradiated ones. The polymer replica can be used during fatigue testing for the determination of the fatigue crack length without unloading or removing the sample from the testing machine. Figure 5 illustrates the 3D measurement of the U-notch of the steel flat tensile specimen used for the localization of the deformation in the defined position at the gauge.
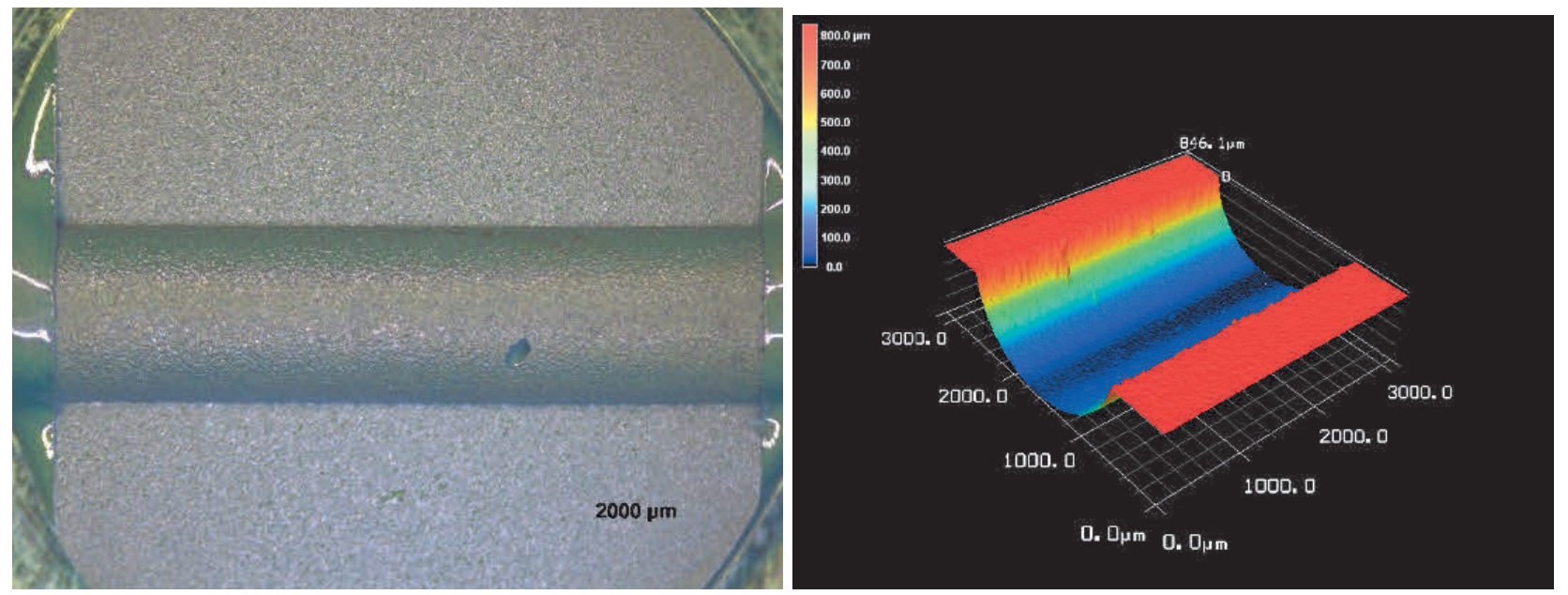

Figure 5 The 3D reconstruction of the U-notch using polymer replica 


\section{CONCLUSION}

The work on the 3D accurate replication of the neutron irradiated materials was recently performed in CVR. The appropriate type of replication polymer matter was chosen from five candidates according to the volume and hardness testing after gamma irradiation. The effect of the radiation dose on the shrinking and embrittlement of the several replicas matters was reported. This selection allows get the replicas with sufficient accuracy in micro-meters. The selected matter was applied to the steel cube with the pre-defined defects (indents) and the accurate replica. The results show that the final accuracy for the replication is $\pm 0.008 \mathrm{~mm}$ for artefacts of $0.1-0.2 \mathrm{~mm}$ size, which is a satisfying result for the volume changes measurements of porous materials.

Another possible application of this type of replication technique were tested. The surface topography of $U$ notch flat tensile specimen was reconstructed using the polymer matter. Moreover, the application of plating upon the polymer replica will allow the scanning electron microscopy observations, e.g. for the fracture analysis. This method can be also used for the fatigue crack length measurement without removing the specimen from the testing machine.

Furthermore, the mechanical replication system was developed and manufactured, and is now successfully used in the SUSEN hot cells.

\section{ACKNOWLEDGEMENTS}

The authors are grateful for the support of the CVR project 18D0013: "IPNOP 3D scanning of irradiated quartz samples". The presented work was financially supported by the Ministry of Education, Youth and Sport Czech Republic - project LQ1603 Research for SUSEN. This work has been realized within the SUSEN Project (established in the framework of the European Regional Development Fund (ERDF) in project CZ.1.05/2.1.00/03.0108 and of the European Strategy Forum on Research Infrastructures (ESFRI) in the project CZ.02.1.01/0.0/0.0/15_008/0000293, which is financially supported by the Ministry of Education, Youth and Sports - project LM2015093 Infrastructure SUSEN.

\section{REFERENCES}

[1] JOHNSTON, A. H. Radiation damage of electronic and optoelectronic devices in space, 2000.

[2] BOURGADE, J.L., COSTLEY, A.E., REICHLE, R., HODGSON, E.R., HSING, W., GLEBOV, V., DECRETON, M., LEEPER, R., LERAY, J.L., DENTAN, M. and HUTTER, T. Diagnostic components in harsh radiation environments: Possible overlap in R\&D requirements of inertial confinement and magnetic fusion systems. Review of Scientific Instruments. 2008. vol. 79, no. 10, p.10F304. 